и венозные тромбоэмболические осложнения: какова взаимосвязь?

\author{
М.Р. Кузнецов ${ }^{1,2}$, О.В. Папышева² \\ ${ }^{1}$ ФГБоу ВО «Российский национальный исследовательский медицинский университет им. Н.И. Пирогова» Министерства \\ здравоохранения Российской Федерации, г. Москва \\ 2 ГБУз «Городская клиническая больница № 29 им. Н.Э. Баумана» Департамента здравоохранения города Москвы, г. Москва
}

\begin{abstract}
Цель обзора: рассмотреть современные данные о связи приема половых гормонов, в том числе средств менопаузальной гормональной терапии (МГТ), с венозными тромбоэмболическими осложнениями (ВТЭ0).

Основные положения. Обсуждается влияние эстрогенов и гестагенов на риск развития венозных тромбозов. Приводятся данные большого наблюдательного исследования, в которое вошли 80396 женщин в возрасте от 40 до 79 лет, зарегистрированных в общеврачебных базах Великобритании и получавших с 1997 по 2017 г. различные виды МГТ. Показано, что сочетание эстрадиола и дидрогестерона по безопасности в отношении ВТЭО значимо не отличается от трансдермальных форм препаратов МГТ, однако в 4-5 раз эффективнее для профилактики сердечно-сосудистых заболеваний (артериальной гипертензии, сахарного диабета и атеросклероза различной локализации). Кроме того, при приеме комбинации эстрадиола с дидрогестероном риск развития венозного тромбоза минимален вне зависимости от режима применения (циклический или постоянный), возраста и индекса массы тела пациенток. Использование ультранизких доз этих препаратов сопровождается меньшим риском тромботических осложнений по сравнению с таковым в контрольной группе. Заключение. Правильный выбор МГТ может способствовать более широкому ее применению, позволяющему максимально снизить вероятность грозных сердечно-сосудистых событий при минимальных рисках ВТЭО.

Ключевые слова: менопаузальная гормональная терапия, тромбофилия, венозные тромбоэмболические осложнения, эстроген, дидрогестерон.
\end{abstract}

Вклад авторов: Кузнецов М.Р. - обзор публикаций по теме статьи, проверка критически важного содержания; Папышева 0.В. - обзор публикаций по теме статьи, анализ и интерпретация данных.

Авторы заявляют об отсутствии возможных конфликтов интересов.

Для цитирования: Кузнецов М.Р., Папышева О.В. Менопаузальная гормональная терапия и венозные тромбоэмболические осложнения: какова взаимосвязь? // Доктор.Ру. 2019. № 11 (166). С. 68-73. DOI: 10.31550/1727-2378-2019-166-11-68-73

\title{
Menopausal Hormonotherapy and Venous Thromboembolic Events: Is There Any Correlation?
}

\author{
M.R. Kuznetsovi, ${ }^{1}$, 0.V. Papysheva² \\ ${ }^{1}$ N.I. Pirogov Russian National Research Medical University; 1 Ostrovityanov Str., Moscow, Russian Federation 117997 \\ ${ }^{2}$ N.E. Bauman Municipal Clinical Hospital No.29; 2 Gospitalnaya pl., Moscow, Russian Federation 111020
}

\begin{abstract}
Objective of the Review: to discuss the available data concerning the correlation between sex hormone administration, including menopausal hormonotherapy (MHT), and venous thromboembolic events (VTEE).

Key Points: the role of estrogens and gestagens in possible venous thrombosis is discussed. The data from a large-scale observational study are presented; the study enrolled 80,396 women aged 40 to 79 years old registered in GP databases of the United Kingdom and treated with various MHT from 1997 to 2017. It is demonstrated that, as far as VTEE is concerned, the safety of a combination of estradiol and didrogesteronis not significantly different from transdermal administration, however it is 4-5 times more efficient in prevention of cardiovascular disorders (arterial hypertension, diabetes mellitus, and atherosclerotic vascular disease). Also, a combination of estradiol and didrogesteron was associated with a minimal risk of venous thrombosis, irrespective of the mode of administration (cyclic or continuous), age, and body mass index of patients. Ultra-low doses of these medications demonstrated lower risk of thrombotic complications vs. controls. Conclusion: the correct choice of MHT can contribute to its wider use, allowing to maximally reduce the risk of severe cardiovascular events with minimal risks of VTEE.

Keywords: menopausal hormonotherapy, thrombophilia, venous thromboembolic events, estrogen, didrogesteron.
\end{abstract}

Contribution: Kuznetsov M.R. - thematic publications reviewing, eview of critically important intellectual material; Papysheva 0.v. — thematic publications reviewing, data analysis and interpretation.

The authors declare that they do not have any conflict of interests.

For reference: Kuznetsov M.R., Papysheva 0.V. Menopausal Hormonotherapy and Venous Thromboembolic Events: Is There Any Correlation? Doctor.Ru. 2019; 11(166): 68-73. DOI: 10.31550/1727-2378-2019-166-11-68-73

Кузнецов Максим Робертович - Ә. м. Н., профессор РАН, профессор кафедры факультетской хирургии ФГБоу ВО «РНИму им. Н.И. Пирогова» Минздрава России; руководитель сосудистого чентра ГБУз «ГКБ № 29 им. Н.Э. Баумана» ДЗМ. 117997, Россия, г. Москва, ул. Островитянова, д. 1. eLIBRARY.RU SPIN: 7146-1348. E-mail: mrkuznetsov@mail.ru

Папышева Ольга Виуленовна - к. м. н., главный врач ГБУз «ГКБ № 29 им. Н.Э. Баумана» ДЗМ. 111020, Россия, г. Москва, Госпитальная пл., д. 2. E-mail: viulen@mail.ru 
0 дной из важных междисциплинарных медицинских проблем считается развитие венозных тромбоэмболических осложнений (ВТЭО). По данным статистических отчетов Министерства здравоохранения Российской Федерации, у нас в стране ежегодно регистрируются около 80000 новых случаев этого заболевания [1]. К ним относят тромбоз глубоких вен (ТГВ), тромбоэмболию легочных артерий (ТЭЛА). Актуальность проблемы ВТЭО связана с их чрезвычайно сильным влиянием на здоровье и жизнь пациентов [2].

С клинических позиций тромботическое поражение венозного русла нижних конечностей наиболее опасно в виде поражения глубоких вен. В пожилом и старческом возрасте частота ТГВ увеличивается в несколько раз и достигает 200 случаев на 100000 человек в год. Легочную эмболию регистрируют ежегодно с частотой 35-40 на 100000 человек.

Следует сказать, что сам по себе тромбоз вен нижних конечностей (глубоких и тем более подкожных) не представляет опасности для жизни пациента (синяя флегмазия или венозная гангрена встречаются крайне редко). Непосредственная угроза жизни больного связана с ТЭЛА. Даже при своевременном выявлении ТГВ от легочной эмболии умирают 6\% пациентов, в то время как частота ТЭЛА может быть и больше, поскольку не всегда диагноз ТГВ ставится вовремя в связи с нечеткой клинической картиной заболевания [3]. В таких случаях ТЭЛА может быть первым проявлением ВТЭО.

Но даже благополучный исход острого периода не означает разрешения проблемы. В отдаленном периоде после ТГВ формируется посттромботическая болезнь нижних конечностей или хроническая тромбоэмболическая легочная гипертензия (ХТЭЛГ). В первом случае происходит глубокая дезорганизация работы венозной системы нижних конечностей с высокой вероятностью развития трофических язв и рецидива венозного тромбоза, а при ХТЭЛГ формируется правожелудочковая недостаточность, напрямую связанная с массивностью поражения легочного русла [4]. Тяжелая ХТЭЛГ в течение 5 лет приводит к смерти 10-15\% больных, перенесших массивную ТЭЛА.

Одной из причин развития ВТЭО следует считать прием гормональных контрацептивов и в меньшей степени средств менопаузальной гормональной терапии (МГТ). МГТ применяется для предупреждения ряда симптомов, беспокоящих многих женщин в период менопаузы, например приливов и ночной потливости. Кроме того, доказана значительная положительная роль МГТ в профилактике остеопороза, при терапии генитоуринарного синдрома; обсуждается польза ее раннего назначения для предотвращения атеросклероза, СД и АГ, что особенно актуально ввиду доминирования сердечно-сосудистых заболеваний среди причин женской смертности.

Риск развития ВТЭО при приеме гормональных препаратов в первую очередь связан с эстрогенами. Обусловлено это тем, что они обладают вазодилатирующим эффектом, приводят к замедлению кровотока в венах [5]. Показано также, что эстрогены увеличивают концентрацию VIII фактора, снижают уровень естественного ингибитора коагуляции - антитромбина III, собственного тромболитического компонента протеина С [6] и усиливают образование тромбина, что отражается в увеличении эндогенного тромбинового потенциала $[7,8]$. Однако и гестагены оказывают существенное влияние на риск венозного тромбоза. Одни из них усугубляют тромботическую активность эстрогенов, другие в этом плане более инертны. Следует сказать, что наиболее безопасными препаратами принято считать те, которые содержат минимальную дозу эстрогенов и максимально безопасный гестаген (табл. 1) [9].
В 2015 г. Национальный институт здравоохранения и совершенствования медицинской помощи Великобритании (National Institute for Health and Care Excellence) опубликовал первые рекомендации по диагностике и лечению симптомов менопаузы в Соединенном Королевстве. Ключевым моментом была необходимость информирования женщин о рисках и преимуществах МГТ с тем, чтобы они могли сделать соответствующий выбор терапии; однако эти рекомендации относятся к применению МГТ в целом, оцениваются различия только между пероральными и трансдермальными формами препаратов [10]. В рекомендациях отмечается необходимость дальнейшего изучения рисков препаратов МГТ, содержащих разные типы прогестагенов в сочетании с эстрогенами.

Подобные рекомендации выпустили и другие организации: Международное общество по менопаузе (2016) [11], Североамериканское общество по менопаузе (2017) [12], Российское общество акушеров-гинекологов (2015) [13]. Они содержат аналогичные положения и подтверждают важность изучения рисков препаратов для МГТ. Рекомендации, вероятно, приведут к росту частоты применения МГТ у женщин с симптомами менопаузы, что повысит потребность в подробных исследованиях отдаленных рисков различных схем МГТ.

Препараты МГТ для приема внутрь могут содержать только эстроген (монотерапия), например конъюгированный лошадиный эстроген или эстрадиол, или эстроген в сочетании с прогестагеном (комбинированная терапия). Из прогестагенов в состав комбинированных препаратов входят медроксипрогестерона ацетат или более новые вещества, такие как норгестрел, дидрогестерон или дроспиренон. Предыдущие исследования, в которых оценивался риск ВТЭ0, сопряженный с использованием различных средств МГТ, либо не делали различий между типами эстрогенов и прогестагенов, либо их мощности хватало только для анализа самых распространенных препаратов [14, 15].

\section{Таблица 1}

Риск развития венозных тромбоэмболических осложнений в зависимости от концентрации эстрогенов и виАа гестагена

\begin{tabular}{|c|c|}
\hline $\begin{array}{c}\text { Комбинированные оральные } \\
\text { контрацептивы (препараты и дозы) }\end{array}$ & $\begin{array}{c}\text { Риск тромбо- } \\
\text { за, отношение } \\
\text { рисков (95\%-ный } \\
\text { доверительный } \\
\text { интервал) }\end{array}$ \\
\hline $\begin{array}{l}50 \text { мкг этинилэстрадиола + } \\
\text { левоноргестрел }\end{array}$ & $5,2(3,4-7,9)$ \\
\hline 30 мкг этинилэстрадиола + дезогестрел & $4,3(3,3-5,6)$ \\
\hline $\begin{array}{l}35 \text { мкг этинилэстрадиола + } \\
\text { ципротерона ацетат }\end{array}$ & $3,9(2,7-5,5)$ \\
\hline $\begin{array}{l}30 \text { мкг этинилэстрадиола + } \\
\text { дроспиренон }\end{array}$ & $3,9(2,7-5,5)$ \\
\hline 30 мкг этинилэстрадиола + гестоден & $3,7(2,8-4,9)$ \\
\hline 20 мкг этинилэстрадиола + дезогестрел & $3,4(2,5-4,6)$ \\
\hline $\begin{array}{l}30 \text { мкг этинилэстрадиола + } \\
\text { левоноргестрел }\end{array}$ & $2,4(1,8-3,2)$ \\
\hline 35 мкг этинилэстрадиола + норгестимат & $2,4(1,7-3,3)$ \\
\hline $\begin{array}{l}20 \text { мкг этинилэстрадиола + } \\
\text { левоноргестрел }\end{array}$ & $2,2(1,3-3,6)$ \\
\hline 20 мкг этинилэстрадиола + гестоден & $2,2(1,4-3,2)$ \\
\hline
\end{tabular}


Результаты рандомизированных контролируемых исследований, обобщенные в Кокрейновском систематическом обзоре [14], основывались главным образом на исследовании Women's Health Initiative, включавшем женщин без хронических соматических заболеваний [16]. В обзоре сказано, что повышенный риск венозных тромбозов сопряжен с приемом пероральных препаратов, содержащих только эстроген, и комбинированных препаратов для приема внутрь с конъюгированным лошадиным эстрогеном и медроксипрогестерона ацетатом.

За прошедшие 20 лет и в других странах появились новые препараты, однако в наблюдательных исследованиях этих препаратов, включенных в метаанализ, применялись различные определения исхода, и мощности этих исследований не хватало для изучения отдельных типов МГТ [15]. Таким образом, информации о риске ВТЭО, сопряженном с конкретными препаратами для МГТ, недостаточно, чтобы сделать информированный выбор препарата для лечения симптомов менопаузы.

В 2019 г. опубликованы интересные данные крупного наблюдательного исследования с участием общей женской популяции Великобритании [17]. В его основе лежал анализ сведений о 80396 женщинах в возрасте от 40 до 79 лет, зарегистрированных в общеврачебных базах и получавших с 1997 по 2017 г. различные виды МГТ. В данном исследовании, помимо прочего, оценивались риски ВТЭО на фоне приема тех или иных гормональных препаратов.

Благодаря большому числу участниц удалось проанализировать множество моментов, ранее не известных в связи со слабой мощностью предыдущих исследований. В частности, показано, что вероятность развития венозных тромбозов ниже при применении трансдермальных форм, чем пероральных. Правда, здесь необходимо остановиться на нескольких моментах.

Во-первых, при назначении МГТ женщинам с расстройствами, обусловленными дефицитом эстрогенов, либо для профилактики остеопороза в первую очередь руководствуются не столько безопасностью, сколько эффективностью препаратов. При этом нужно учитывать, что МГТ, помимо купирования симптомов, при наличии показаний к назначению снижает смертность от ИБС и общую смертность. Иметь в виду эти дополнительные эффекты крайне важно, т.к. именно сердечно-сосудистые заболевания являются основной причиной инвалидизации и смертности женщин в постменопаузе $[11,18]$. Выявлено также, что ранняя менопауза увеличивает риск АГ и инфаркта миокарда в 2 раза, а овариэктомия до 50 лет повышает частоту сердечно-сосудистых заболеваний в 4,55 раза [19].
Снижение смертности от ИБС и профилактика сердечно-сосудистых заболеваний обусловлены влиянием МГТ на липидный спектр крови и зависят от типа гормонов (эстрогена и прогестерона), дозы и пути введения. Доказано, что оральные эстрогены оказывают более выраженное положительное влияние на липидный спектр крови, т.к. при первичном прохождении через печень кривая концентрации гормонов в 4-5 раз выше, чем при парентеральном пути введения. В итоге проявляется это тем, что повышается элиминация из крови атерогенных ЛПнП, снижается индекс атерогенности и тормозится развитие атеросклероза за счет индуцированного эстрогенами увеличения на поверхности гепатоцитов количества рецепторов к лПнП [20].

С этих позиций крайне интересны выпущенные в 2018 г. новые рекомендации по менопаузе и СД, в которых говорится, что МГТ дает положительный эффект на гликемический профиль у женщин без СД и с СД 2 типа. Кроме того, отмечается, что своевременно начатая МГТ может отсрочить развитие СД у женщин в постменопаузе.

Наиболее важным представляется то, что именно пероральная МГТ предпочтительна для профилактики развития СД, а из доступных и максимально исследованных гестагенов в составе пероральной МГТ наиболее оправдано использование прогестерона и дидрогестерона [21].

Возвращаясь к ВТЭ0, следует сказать, что, несмотря на выводы ряда исследований о большей безопасности трансдермальных форм препаратов, многие авторы отмечают, что пероральные формы тоже существенно различаются между собой в зависимости от вида эстрогена, его дозировки, а также выбора гестагена. В целом прием препаратов, содержащих конъюгированный лошадиный эстроген, сопряжен с более высоким риском по сравнению с таковым препаратов с эстрадиолом (Е2). Наибольший риск развития ВТЭО связан с применением конъюгированного лошадиного эстрогена с медроксипрогестерона ацетатом (рис. 1).

С другой стороны, комбинация эстрадиола с дидрогестероном характеризовалась минимальным риском венозных тромбозов, статистически значимо не отличавшимся от риска при использовании трансдермальных форм. Сочетания эстрадиола с дроспереноном или норгестрелом отнесли в одну группу, поскольку пациенток, принимавших МГТ с этими гестагенами, было недостаточно для отдельного статистического анализа. Авторы замечают, что в Великобритании менее $1 \%$ женщин принимают эти препараты из-за с высокого риска тромботических осложнений [17].

Был отмечен еще один важный момент. При применении эстрадиола с дидрогестероном риск венозных тромбозов

\footnotetext{
Рис. 1. Риск развития венозных тромбоэмболических осложнений в зависимости от вида эстрогена

и гестагена [17], отношение шансов (95\%-ный доверительный интервал).

Ппимечание. Здесь и в рисунках 3, 4: КАЭ- конъюгированньгй лошадиный эстроген
}

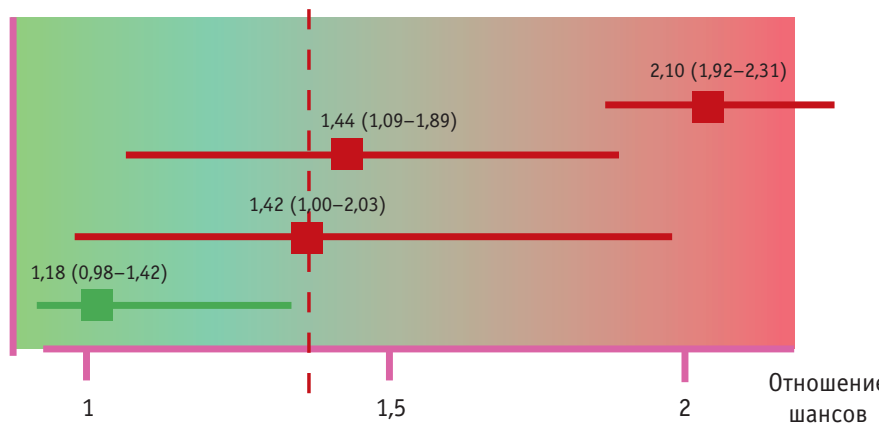

КЛЭ + медроксипрогестерона ацетат

Е2 + медроксипрогестерона ацетат

Е2 + дроспиренон/норгестрел

E2 + дидрогестерон 
не возрастал ни при изменении режима терапии (циклический или непрерывный), ни при увеличении возраста пациенток, ни при повышении их ИМТ (рис. 2-4).

Последнее особенно актуально, поскольку пожилой возраст и избыточная масса тела сами по себе являются отдельными независимыми и усиливающими друг друга тромбофилическими состояниями, существенно повышающими риск развития ВТЭО.

Тромбофилии могут быть как врожденными (табл. 2), так и приобретенными.

Приобретенные или вторичные тромбофилии:

- активный рак (включая миелопролиферативные и миелодиспластические заболевания);

- наличие антифосфолипидных антител в достаточном титре (волчаночных, антикардиолипиновых, анти- $\beta 2$ и др.);

- аутоиммунные нарушения;

- гепарин-индуцированная тромбоцитопения;

- ночная пароксизмальная гемоглобинурия;

- инфекция (пневмония, сепсис, инфекция мочевых путей, ВИЧ);
- гипергомоцистеинемии;

- тромботическая тромбоцитопеническая пурпура;

- дислипидемия, ожирение;

- микроальбуминурия, нефротический синдром;

- обезвоживание;

- синдром диссеминированного внутрисосудистого свертывания;

- беременность, послеродовой период;

- химиотерапия;

- терапия эстрогенами или прогестероном;

- прием селективных модуляторов рецепторов эстрогена (тамоксифена и ралоксифена).

Среди врожденных тромбофилий [22] выделяют семь наиболее злокачественных (см. табл. 2). Однако если еще несколько лет назад сам по себе термин «тромбофилия» вызывал чрезмерную озабоченность клиницистов, приводящую к необоснованному длительному, а часто пожизненному, назначению антикоагулянтов, то в настоящее время подход значительно изменился. Кроме того, только гомозиготные врожденные тромбофилии несут существенный

Рис. 2. Риск венозных тромбоэмболических осложнений в зависимости от режима терапии и Аозировки эстрадиола с Аилрогестероном [17], отношение шансов (95\%-ный Аоверительный интервац)

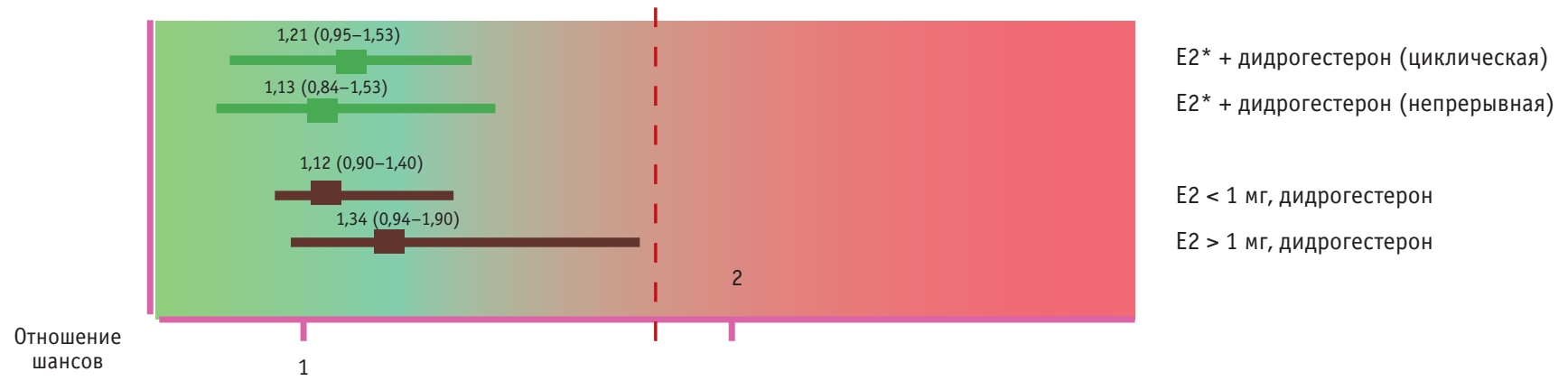

Рис. 3. Риск венозных тромбоэмболических осложнений в зависимости от возраста пациенток [17], отношение шансов (95\%-ный доверительный интервац)

40-54 года

$$
\begin{aligned}
& \text { Е2 + дидрогестерон } \\
& \text { Е2 + медроксипрогестерона ацетат } \\
& \text { Е2 + норэтистерон } \\
& \text { КЛЭ + медроксипрогестерона ацетат }
\end{aligned}
$$

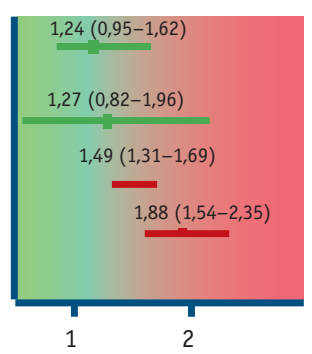

55-64 года

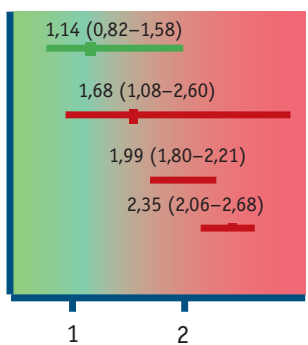

65-79 лет

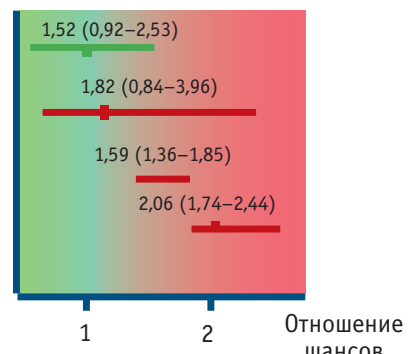

Рис. 4. Риск венозных тромбоэмболических осложнений в зависимости от индекса массы тела (ИМТ) [17], отношение шансов (95\%-ный доверительный интервац)
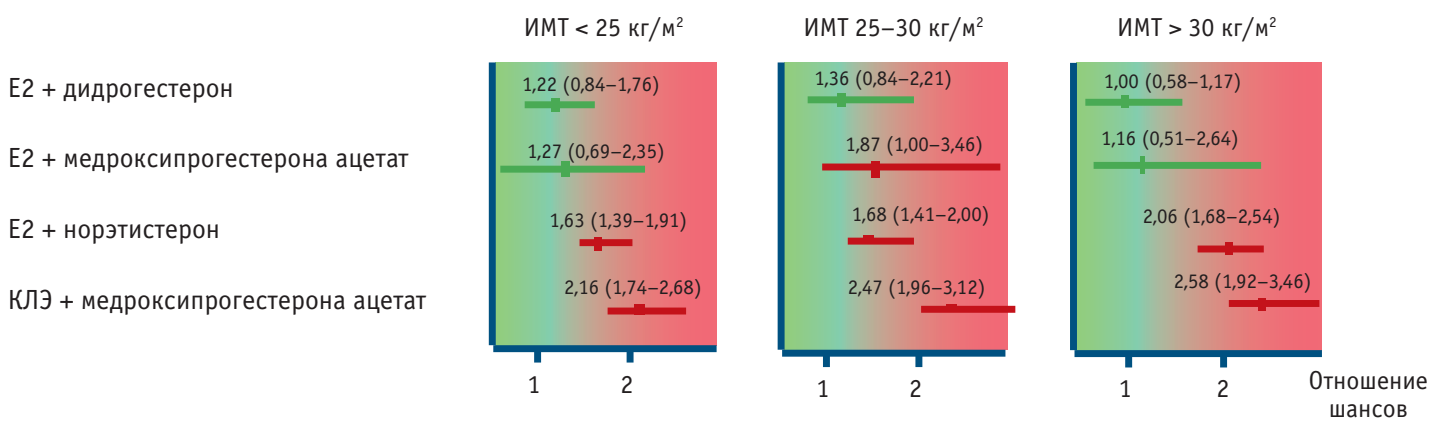
Таблица 2 ]

Современная кмассификация тромбофимий. ВрожАенные (семейные и первичные) тромбофилии

\begin{tabular}{|c|c|}
\hline Причины тромбофилии & $\begin{array}{c}\text { Уровень } \\
\text { доказательности }\end{array}$ \\
\hline Дефицит антитромбина III & \multirow{7}{*}{$\begin{array}{l}\text { Безусловно } \\
\text { подтвержденные } \\
\text { данные }\end{array}$} \\
\hline Дефицит протеина С & \\
\hline Дефицит протеина S & \\
\hline $\begin{array}{l}\text { Резистентность фактора Va } \\
\text { к активированному протеину C }\end{array}$ & \\
\hline Мутация фактора V Лейден & \\
\hline Мутация протромбина (20210А) & \\
\hline $\begin{array}{l}\text { Гомоцистинурия, } \\
\text { гипергомоцистеинемия — патология } \\
\text { метаболизма метионина }\end{array}$ & \\
\hline $\begin{array}{l}\text { Повышение активности фибриногена, } \\
\text { факторов II, VIII, IX, XI }\end{array}$ & \multirow[t]{4}{*}{$\begin{array}{l}\text { Подтвержденные } \\
\text { данные }\end{array}$} \\
\hline Дисфибриногенемия & \\
\hline $\begin{array}{l}\text { Гипоплазминогенемия } \\
\text { и дисплазминогенемия }\end{array}$ & \\
\hline Серповидно-клеточная анемия & \\
\hline $\begin{array}{l}\text { Снижение уровней витамин } \\
\text { К-зависимого белка Z и Z-зависимого } \\
\text { ингибитора }\end{array}$ & \multirow[t]{4}{*}{\begin{tabular}{|l} 
Слабо \\
подтвержденные \\
данные
\end{tabular}} \\
\hline $\begin{array}{l}\text { Снижение уровня ингибитора пути } \\
\text { тканевого фактора (TFPI) }\end{array}$ & \\
\hline $\begin{array}{l}\text { Дефицит тканевого активатора } \\
\text { плазминогена (t-PA) }\end{array}$ & \\
\hline $\begin{array}{l}\text { Высокий уровень ингибитора актива- } \\
\text { тора плазминогена } 1 \text { типа (PAJ-1) }\end{array}$ & \\
\hline Полиморфизм фактора XIII (Val34Leu) & \multirow{2}{*}{$\begin{array}{l}\text { Нет } \\
\text { подтверждающих } \\
\text { данных }\end{array}$} \\
\hline $\begin{array}{l}\text { Повышенный уровень } \\
\text { активированного тромбином } \\
\text { ингибитора фибринолиза (TAFI) }\end{array}$ & \\
\hline
\end{tabular}

риск тромботических осложнений, а при гетерозиготных риск практически не отличается от среднестатистического в популяции.

Тем не менее следует признать, что нет возможности каким-либо образом повлиять на врожденные тромбофилии, и приходится в какой-то степени с ними «мириться». Сложнее дело обстоит с некоторыми приобретенными тромбофилиями, которые могут протекать скрыто и накапливаться в течение жизни. С другой стороны, при правильном подходе можно минимизировать или полностью устранить риск ВТЭО, обусловленный приобретенными тромбофилическими состояниями.

Одной из таких тромбофилий, помимо возраста и избыточной массы тела, является обезвоживание. В некоторых

\section{ЛИТЕРАTУРA / REFERENCES}

1. Андрияшкин А.В., Андрияшкин В.В., Арутюнов Г.П., Баринов В.Е., Бицадзе B.О., Бодыхов М.К. и др. Российские клинические рекомендации по диагностике, лечению и профилактике венозных тромбоэмболических осложнений (ВТЭО). Флебология. 2015; 4(2). 52 c. [Andriyashkin A.V., Andriyashkin V.V., Arutyunov G.P., Barinov V.E., Bitsadze V.O., Bodykhov M.K. i dr. Rossiiskie klinicheskie rekomendatsii po diagnostike, lecheniyu i profilaktike venoznykh tromboembolicheskikh oslozhnenii (VTEO). Flebologiya. 2015; 4(2). 52 s. (in Russian)] комбинированных пероральных препаратах, помимо эстрогенов, содержатся гестагены, обладающие выраженным тромбогенным эффектом, - медроксипрогестерона ацетат [19] и дроспиренон [23, 24]. Последний усиливает агрегацию тромбоцитов, что может быть причиной выраженного тромбогенного эффекта [25]. Дидрогестерон в этом плане представляется инертным, что выделяет его из всех гестагенов как препарат с минимальным риском развития ВТЭО.

Более того, некоторые авторы отмечают, что ультранизкодозированная МГТ с дидрогестероном в своем составе (0,5 мг 17 $\beta$-эстрадиола + 2,5 мг дидрогестерона) приводит даже к снижению риска ВТЭО по сравнению с отсутствием МГТ за счет статистически значимого уменьшения на 16\% содержания антигена ингибитора активатора плазминогена 1 и торможения тем самым тромбогенной активности [26].

\section{ЗАКЛЮЧЕНИЕ}

В настоящее время практически всеми специалистами признается важнейшая роль менопаузальной гормональной терапии (МГТ) не только в купировании симптомов дефицита эстрогенов у женщин в постенопаузе, но и в профилактике грозных осложнений у этих женщин при назначении МГТ по показаниям. Чаще всего подобные осложнения связаны с сердечно-сосудистыми заболеваниями, представляющими наибольшую опасность для жизни женщин в этом возрастном периоде. Однако осложнения, которые могут быть связаны с приемом половых гормонов, в первую очередь венозные тромбозы, нередко ограничивают применение заместительной терапии. В связи с этим ведутся исследования, направленные на снижение риска венозных тромбоэмболических осложнений (ВТЭО) при использовании различных препаратов МГТ, и достаточно часто появляются суждения, что трансдермальные формы гораздо безопаснее.

Однако и эффективность этих препаратов в профилактике СД и атеросклероза значительно ниже, чем пероральных форм. При этом соотношение пользы и риска при применении оральных препаратов во многом зависит от дозировки и вида эстрогена, а также гестагеновой составляющей.

Наибольший риск развития ВТЭО связан с использованием конъюгированного лошадиного эстрогена и более высоких доз эстрадиола. Целый ряд гестагенов, входящих в комбинированные препараты, может дополнительно стимулировать эту тромбогенную активность, что и вызывает негативное суждение о пероральной МГТ.

Но прием комбинации эстрадиола с дидрогестероном, обладающей выраженной защитной функцией от сердечно-сосудистых заболеваний, сопровождается минимальным риском ВТЭ0, сравнимым с риском трансдермальных форм гормональных препаратов. Кроме того, риск развития ВТЭО может снижать ультранизкодозированная комбинация данных компонентов.

Следует признать, что правильный выбор МГТ может способствовать более широкому ее применению, позволяющему максимально снизить вероятность грозных сердечнососудистых событий при минимальных рисках ВТЭО.

2. García Sabrido J.L., Pacheco Sánchez D. Profilaxis de la enfermedad tromboembolica perioperatoria en cirugta general. Cir. Esp. 2001; 69: 49-55.

3. Duranteau J., Taccone F.S., Verhamme P., Ageno W.; ESA VTE Guidelines Task Force. European guidelines on perioperative venous thromboembolism prophylaxis: Intensive care. Eur. J. Anaesthesiol. 2018; 35(2): 142-6. DOI: 10.1097/EJA.0000000000000707

4. Al Yami M.S., Silva M.A., Donovan J.L., Kanaan A.O. Venous thromboembolism prophylaxis in medically ill patients: a mixed 
treatment comparison meta-analysis. J. Thromb. Thrombolysis. 2018; 45(1): 36-47. D0I: 10.1007/s11239-017-1562-5

5. Ceballos C., Ribes C., Amado J.A., Pérez J., García Unzueta M.T., de Berrazueta J.R. Venous endothelial function in postmenopausal women who are receiving long-term estrogen and progestagen therapy. Fertil. Steril. 2000; 74(2): 268-73. DOI: 10.1016/s00150282(00)00627-0

6. Salobir B.G., Keber J., Vrabic L. A randomized, placebo-controlled trial of the effects of continuous combined hormone replacement therapy on coagulation and fibrinolytic systems in healthy postmenopausal women. Fertil. Steril. 2002; 78(6): 1178-83. D0I: 10.1016/s00150282(02)04291-7

7. Scarabin P.Y., Hemker H.C., Clément C., Soisson V., Alhenc-Gelas M. Increaset thrombin generation among postmenopausal women using hormone therapy: importance of the route of estrogen administration and progestagens. Menopause. 2011; 18(8): 873-9. DOI: $10.1097 /$ gme.0b013e31820eee88

8. Teede H.J., McGrath B.P., Smolich J.J., Malan E., Kotsopoulos D., Liang Y.L. et al. Postmenopausal hormone replacement therapy increaces coagulation activity and fibrinolysis. Arterioscler. Thromb. Vasc. Biol. 2000; 20(5): 1404-9. DOI: 10.1161/01.atv.20.5.1404

9. Bastos M., Stegeman B.H., Rosendaal F.R., Van Hylckama Vlieg A. Helmerhorst F.M., Stijnen T. et al. Combined oral contraceptives: venous thrombosis. Cochrane Database Syst. Rev. 2014; 3: CD010813. DOI: 10.1002/14651858.CD010813.pub2

10. Lumsden M.A., Davies M., Sarri G.; Guideline Development Group for Menopause: Diagnosis and Management (NICE Clinical Guideline No. 23). Diagnosis and Management of Menopause: The National Institute of Health and Care Excellence (NICE) Guideline. JAMA Intern. Med. 2016; 176(8): 1205-6. DOI: 10.1001/jamainternmed.2016.2761

11. Baber R.J. Panay N., Fenton A.; IMS Writing Group. IMS Recommendations on women's midlife health and menopause hormone therapy. Climacteric. 2016; 19(2): 109-50. DOI: 10.3109/13697137.2015.1129166

12. The 2017 hormone therapy position statement of The North American Menopause Society. Menopause. 2018; 24(7): 728-53. DOI: 10.1097/GME.0000000000001241

13. Российское общество акушеров-гинекологов, Российская ассоциация по менопаузе. Менопауза и климактерическое состояние у женщины. Клинические рекомендации. РОАГ: КР117; 2016. 45 c. [Rossiiskoe obshchestvo akusherov-ginekologov, Rossiiskaya assotsiatsiya po menopauze. Menopauza i klimaktericheskoe sostoyanie u zhenshchiny. Klinicheskie rekomendatsii. ROAG: KR117; 2016. 45 s. (in Russian)]

14. Marjoribanks J., Farquhar C., Roberts H., Lethaby A., Lee J. Longterm hormone therapy for perimenopausal and postmenopausal women. Cochrane Database Syst. Rev. 2017; 1: CD004143. DOI: 10.1002/14651858.CD004143.pub5
15. Mohammed K., Abu Dabrh A.M., Benkhadra K., Al Nofal A., Carranza Leon B.G., Prokop L.J. et al. Oral vs transdermal estrogen therapy and vascular events: a systematic review and metaanalysis. J. Clin. Endocrinol. Metab. 2015; 100(11): 4012-20. DOI: 10.1210/ jc.2015-2237

16. Manson J.E., Chlebowski R.T., Stefanick M.L., Aragaki A.K., Rossouw J.E., Prentice R.L. et al. Menopausal hormone therapy and health outcomes during the intervention and extended poststopping phases of the Women's Health Initiative randomized trials. JAMA. 2013; 310(13): 1353-68. DOI: 10.1001/jama.2013.278040

17. Vinogradova Y., Coupland C., Hippisley-Cox J. Use of hormone replacement therapy and risk of venous thromboembolism: nested case-control studies using the QResearch and CPRD databases. BMJ. 2019; 364: k4810. DOI: 10.1136/bmj.k4810

18. Савельева Г.М., Сухих Г.Т., Серов В.Н., Радзинский В.Е., Манухин И.Б., ред. Гинекология: национальное руководство. М.: ГЭОТАP-Meдua; 2017. 1008 c. [Savel'eva G.M., Sukhikh G.T., Serov V.N., Radzinskii V.E., Manukhin I.B., red. Ginekologiya: natsional'noe rukovodstvo. M.: GEOTAR-Media; 2017. 1008 s. (in Russian)]

19. Atsma F., Bartelink M.L., Grobbee D.E., van der Schouw Y.T. Postmenopausal status and early menopause as independent risk factors for cardiovascular disease: a meta-analysis. Menopause. 2006; 13(2): 265-79. D0I: 10.1097/01.gme.0000218683.97338.ea

20. Lobo R.A., Bush T., Carr B.R., Pickar J.H. Effects of lower doses of conjugated equine estrogens and medroxyprogesterone acetate on plasma lipids and lipoproteins, coagulation factors, and carbohydrate metabolism. Fertil. Steril. 2001; 76(1): 13-24. D0I: 10.1016/ s0015-0282(01)01829-5

21. Slopiena R. Wender-Ozegowskab E., Rogowicz-Frontczakcet A., Meczekalski B., Zozulinska-Ziolkiewicz D., Jaremek J.D. et al. Menopause and diabetes: EMAS clinical guide. Maturitas. 2018; 117: 6-10. DOI: 10.1016/j.maturitas.2018.08.009

22. Kitchens C.S., Konkle B.A., Consultative C.M. Hemostasis and thrombosis. Philadelphia: Saunders; 2013. 840 p.

23. Madigan D., Shin J. Drospirenone-containing oral contraceptives and venous thromboembolism: an analysis of the FAERS database. Open Access J. Contracept. 2018; 9: 29-32. DOI: 10.2147/OAJC. S161737

24. Карева Е.Н. Тромбозы и гестагены. Доктор.Ру. 2019; 7(162): 57-64. [Kareva E.N. Trombozy $i$ gestageny. Doktor.Ru. 2019; 7(162): 57-64. (in Russian)]

25. Fan X., Chen X., Wang C., Dai J., Lu Y., Wang K. et al. Drospirenone enhances GPIb-IX-V-mediated platelet activation. J. Thromb. Haemost. 2015; 13(10): 1918-24. DOI: 10.1111/jth.13109

26. Piroga M., Jacha R., Undas A. Effects of ultra-low-dose versus standard hormone therapy on fibrinolysis and thrombin generation in postmenopausal women. Eur. J. Obstet. Gynecol. Reprod. Biol. 2017; 217: 77-82. DOI: 10.1016/j.ejogrb.2017.08.023

\section{СПИСОК СОКРАЩЕНИЙ}

$\begin{array}{ll}\text { АГ } & \text { - артериальная гипертензия } \\ \text { АД } & \text { - артериальное давление } \\ \text { АЛТ } & \text { - аланинаминотрансфераза } \\ \text { АМФ } & \text { - аденозинмонофосфат } \\ \text { АСТ } & \text { - аспартатаминотрансфераза } \\ \text { АТФ } & \text { - аденозинтрифосфат } \\ \text { ВИЧ } & \text { - вирус иммунодефицита человека } \\ \text { ВОЗ } & \text { - Всемирная организация здравоохранения } \\ \text { ВПЧ } & \text { - вирус папилломы человека } \\ \text { ВРТ } & \text { - вспомогательные репродуктивные технологии } \\ \text { ГНРГ } & \text { - гонадотропин-рилизинг-гормон } \\ \text { ДНК } & \text { - дезоксирибонуклеиновая кислота } \\ \text { ЖКТ } & \text { - желудочно-кишечный тракт } \\ \text { ИБС } & \text { - ишемическая болезнь сердца } \\ \text { ИМТ } & \text { - индекс массы тела } \\ \text { ИППП } & \text { - инфекции, передаваемые половым путем } \\ \text { ИФА } & \text { - иммуноферментный анализ }\end{array}$

\begin{tabular}{|c|c|}
\hline ПГ & — лютеинизурующий гормон \\
\hline ЛпвП & - липопротеиды высокой плотности \\
\hline ЛПНП & - липопротеиды низкой плотности \\
\hline 5-10 & $\begin{array}{l}\text { - Международная классификация болезне } \\
\text { 10-го пересмотра }\end{array}$ \\
\hline ИPT & $\begin{array}{c}\text { - магнитно-резонансная томография, } \\
\text { магнитно-резонансная томограмма }\end{array}$ \\
\hline 7ЦР & - полимеразная цепная реакция \\
\hline СД & - сахарный диабет \\
\hline 4 & - ультразвуковое исследование \\
\hline DCГ & — фолликулостимулирующий гормон \\
\hline $\mathrm{HC}$ & - центральная нервная система \\
\hline & - экстракорпоральное оплодотворение \\
\hline & - С-реактивный белок \\
\hline OMA & $\begin{array}{c}\text { - индекс инсулинорезистентности } \\
\text { (homeostasis model assessment) }\end{array}$ \\
\hline & - иммуноглобулин \\
\hline
\end{tabular}

